\title{
Modelo de Recomendación Personalizada en Cursos Virtuales basado en Computación Ubicua y Agentes Inteligentes
}

\author{
Demetrio A. Ovalle (1), Oscar M. Salazar(1), Néstor D. Duque(2) \\ (1) Universidad Nacional de Colombia, Facultad de Minas, Departamento de Ciencias de la Computación y \\ la Decisión, Grupo de Investigación GIDIA, Medellín-Colombia (\{dovalle,omsalazaro\}@unal.edu.co) \\ (2) Universidad Nacional de Colombia, Facultad de Administración, Departamento de Informática y \\ Computación, Grupo de Investigación GAIA, Manizales-Colombia (ndduqueme@unal.edu.co)
}

Recibido Dic. 16, 2013; Aceptado Feb. 21, 2014; Versión final recibida Mar. 17, 2014

\begin{abstract}
Resumen
El objetivo de este artículo es proponer un modelo de recomendación personalizada de recursos educativos para cursos virtuales adaptativos que incorpora las bondades de la computación ubicua y de los agentes inteligentes. Dicho modelo busca proveer de información relevante y personalizada a los estudiantes sobre la planificación de sus cursos virtuales, evaluación en línea, búsqueda y recuperación de objetos de aprendizaje. La metodología utilizada para la construcción del sistema es MAS-CommonKADS la cual ofrece modelos útiles para las fases de conceptualización, análisis y diseño expresados a través de los artefactos que proporciona la extensión del Lenguaje Unificado de Modelado para Agentes (AUML). EI prototipo construido exhibe agentes inteligentes proactivos y deliberativos que permiten la búsqueda y recomendación de información adaptada al perfil del estudiante. Para validar el sistema se realizó una fase de pruebas con un escenario de aprendizaje, el cual evidencia la eficacia de utilizar este tipo de tecnologías en entornos virtuales y ubicuos de aprendizaje.
\end{abstract}

Palabras clave: computación ubicua, cursos virtuales adaptativos (CVAs), agentes inteligentes, ontologías

\section{Customized Recommendation Model for Virtual Courses based on Ubiquitous Computing and Intelligent Agents}

\begin{abstract}
The aim of this paper is to propose a customized recommendation model of educational resources for virtual courses that incorporates the benefits of ubiquitous computing and intelligent agents. This model is intended to provide relevant and personalized information to students about their virtual courses planning, online assessment, search and retrieval of learning objects. The methodology used for the construction of the system is MAS-CommonKADS which offers useful models for the phases of conceptualization, analysis and design expressed through artifacts provided by the Agent Unified Modeling Language (AUML). The prototype developed exhibits intelligent proactive and deliberative agents that allow the search and recommendation of information adapted to the student's profile. To validate the system a testing phase on a learning scenario was performed which demonstrate the effectiveness of using this kind of technology within ubiquitous virtual learning environments.
\end{abstract}

Keywords: ubiquitous computing, adaptive virtual courses (AVC), intelligent agents, ontologies 


\section{INTRODUCCIÓN}

Múltiples autores han discutido acerca de los métodos de enseñanza-aprendizaje tradicionales, dependientes de las aulas físicas y totalmente magistrales, concluyendo que las clases son muy artificiales, pasivas, rígidas y no responden con claridad a las necesidades que la sociedad está planteando con respecto a los procesos de enseñanza-aprendizaje (Acevedo et al., 2010; Kinshuk y Graf, 2012). Lo anterior se debe a que dichos métodos de enseñanza no son personalizados, es decir, no están orientados a las necesidades, gustos y técnicas de aprendizaje de cada uno de los estudiantes lo que genera desinterés por parte de éstos y retrasa notoriamente el proceso de aprendizaje. Estas problemáticas, sumadas a las teorías modernas que sostienen que "en el proceso de aprendizaje los estudiantes no absorben de forma pasiva el conocimiento personalmente significativo, sino que más bien lo crean de forma activa, a partir de su experiencia del mundo" (Vojak, 2011), reflejan las necesidades del mundo actual con respecto al planteamiento de nuevos mecanismos de enseñanza-aprendizaje que estén más orientados al entorno en el que se desenvuelven los estudiantes, al igual que a las características que describen sus gustos y necesidades.

El crecimiento de la información digital sumado al auge en las telecomunicaciones de alta velocidad y la creación de sistemas ubicuos inteligentes (Charitonos et al., 2012; Gómez et al., 2014) brindan herramientas para el desarrollo de sistemas de recomendación personalizados enfocados a dispositivos móviles, dando paso a un nuevo paradigma en donde los usuarios cuentan con una gran gama de interfaces y dispositivos para comunicarse con los sistemas de información, y en donde el contexto juega un papel sumamente importante. Para ello se está haciendo uso de tecnologías y enfoques novedosos como agentes de software inteligentes, dispositivos inalámbricos, buscadores de información adaptativos y personalizados con el fin de crear modelos de recomendación computarizados. El objetivo de los dispositivos móviles y la computación ubicua es ayudar al usuario en el cumplimiento de sus tareas cotidianas sin atentar a su privacidad y ofrecer interfaces de interacción hombre-máquina que sean amigables y fáciles de utilizar (Kinshuk et al., 2012). La computación ubicua debe exhibir de igual manera características de proactividad y adaptatividad de acuerdo al contexto en el cual se desenvuelve el usuario.

Por su parte, los Cursos Virtuales Adaptativos (CVAs) son herramientas computacionales capaces de guiar al alumno a lo largo de un dominio particular del conocimiento, resolviendo durante el proceso tareas tales como la elaboración de una estrategia de planificación de actividades de aprendizaje, adaptación de contenidos educacionales y evaluación de su desempeño durante el desarrollo de un curso virtual (Duque y Ovalle, 2011). Cabe señalar que los contenidos educacionales más utilizados en la actualidad en el marco de los CVAs son los Objetos de Aprendizaje (OAs), que se definen como entidades auto descritas mediante metadatos bien definidos cuyo principal objetivo es permitir su almacenaje y recuperación por tantos usuarios como sea posible (Raju y Ahmed, 2012). De igual manera los metadatos reflejan la finalidad para la cual fue creada el objeto y hacia qué población o dominio está enfocado. Con el objetivo de estandarizar los esquemas de representación de metadatos de OAs se han desarrollado numerosos trabajos, uno de los más reconocidos es el estándar IEEE-LOM (Primo et al., 2012), el cual fue utilizado para el desarrollo de esta investigación.

Con el fin de realizar sistemas de recomendación de OAs se utiliza el concepto de ontología la cual trabaja sobre el dominio específico de conocimientos del CVA y aplica un método para obtener una representación formal de los conceptos del dominio y las relaciones que existen entre estos (Tramullas et al., 2013). Existen diferentes lenguajes para representar ontologías, el más utilizado es OWL (Meditskos y Bassiliades, 2008), el objetivo principal de este lenguaje es el procesamiento automático de información por parte de las aplicaciones en la web, en vez de que sea procesada con intervención humana. Desde esta perspectiva, surge el concepto de la Web Semántica, que se define según (Berners-Lee et al., 2001) como "una extensión de la web actual en donde la información tiene un significado bien definido, es más entendible por los computadores y en donde las personas pueden trabajar cooperativa y colaborativamente". A partir de este nuevo paradigma, las ontologías aparecen como la vía para representar el conocimiento de la Web de forma que se haga legible y reutilizable por los computadores (Tramullas et al., 2013).

Los Sistemas Multi-Agente (SMA) provenientes de la inteligencia artificial distribuida, se definen como sistemas complejos constituidos por agentes autónomos con conocimientos específicos en un dominio, capaces de interactuar para realizar tareas orientadas a la consecución de un objetivo común (Shoham y Leyton-Brown, 2008; Wooldridge, 2009). Otra de las características de este tipo de sistemas es la facilidad de adquisición y procesamiento de información que se encuentra altamente distribuida, lo que se complementa perfectamente con la computación ubicua y los dispositivos móviles. Los agentes móviles por su parte (Liu y Jiang, 2014) son procesos capaces de moverse a través de una red informática, ya sea LAN (Local Área Network) o WAN (Wide Area Network), migrando o clonando su código y estado de una máquina a otra. Interactuando con dispositivos extraños, recopilando información para luego volver a su 
origen con los datos obtenidos. En efecto, es mucho más eficiente que un agente se movilice hasta una ubicación remota y haga una búsqueda, a traer toda la información para luego ser procesada y filtrada.

El objetivo de este artículo es presentar el análisis, diseño e implementación de un SMA ubicuo el cual pretende entregar al estudiante información filtrada y adaptada a su perfil referente a los CVAs. Esta información tiene como finalidad, el guiar al estudiante dentro de los cursos mediante la planificación de cursos virtuales adaptativos, evaluación en línea, búsqueda y recuperación de objetos de aprendizaje, temáticas y de tutores especializados, utilizando dispositivos móviles. Para la construcción del SMA ubicuo se utilizaron los modelos propuestos en las fases de análisis y diseño de la metodología MASCommonKADS (Hasan y Isaac, 2011) a través de los diferentes artefactos que proporciona AUML (extensión de UML para agentes). La fase de implementación propia del SMA se llevó a cabo utilizando las herramientas como el framework JADE (Bordini et al., 2006) y Protégé (Tudorache et al., 2013) que permitió el desarrollo de la ontología referente al dominio de aplicación.

\section{TRABAJOS RELACIONADOS}

Esta sección presenta algunos trabajos de investigación sobre modelos y sistemas de aprendizaje basados en tecnologías tales como ambientes de aprendizaje ubicuo, sensible al contexto, basado en ontologías y objetos de aprendizaje, así como en agentes inteligentes y redes de sensores inalámbricos.

CAULS es el nombre de un sistema de aprendizaje ubicuo y sensible al contexto basado en tecnología de RFID, sensores inalámbricos, dispositivos portátiles embebidos y tecnologías de bases de datos para detectar y examinar los comportamientos de los estudiantes en un museo en Taiwan propuesto por (Chen et al., 2012). Este trabajo planteó un cuestionario que se solucionaba con información del contexto en el que se desenvolvía el estudiante y proporcionada por el sistema. Los resultados arrojados por la aplicación del cuestionario, arrojaron puntuaciones con mejoría significativa, lo que indica la eficacia del uso de este tipo de tecnologías en ambientes de aprendizaje moderno.

Un ambiente de aprendizaje sensible al contexto fue desarrollado por Hwang et al (2009) para guiar a los investigadores novatos por medio de prácticas presenciales en procesos de difracción de rayos $X$ en monocristal. El dominio de aplicación de esta investigación corresponde a experimentos científicos y por lo tanto, cuando un estudiante entra a un laboratorio y se encuentra en frente a un instrumento, los sensores son capaces de detectar la ubicación del estudiante y de esta forma transfieren la información al servidor. Mediante el análisis en tiempo real de los contextos ambientales/personales, el perfil y el portafolio en línea de los estudiantes, el sistema de aprendizaje es capaz de orientar a los estudiantes a desenvolverse en el mundo real mostrándoles la información relevante en el momento oportuno, los procesos a aplicar, las normas que rigen en el laboratorio y los procedimientos de manejo de emergencias. Los resultados experimentales mostraron los beneficios de aplicar el enfoque de aprendizaje ubicuo y sensible al contexto en ciencias, así como el ahorro de mano de obra para asistir y monitorear a los estudiantes. También este sistema innovador puede ser aplicado a los experimentos de otras ciencias como química o biotecnología, destinado a egresados en ciencias, estudiantes de doctorado en las universidades e incluso para trabajadores de institutos de investigación. Otro trabajo afín a éste es el propuesto por (Wang et al., 2012), el cual incorpora tecnologías de sensibilidad al contexto y sistemas de recomendación basados en filtrado colaborativo para el desarrollo de cursos estandarizados y adaptativos.

(Primo et al., 2013) analizan el uso de técnicas de la Web semántica para la recomendación de OAs. A partir de la exploración de dichas técnicas se propone un modelo basado en ontologías para la representación de metadatos. El conocimiento plasmado en la ontología es almacenado en un archivo OWL, lo cual le otorga características de interoperabilidad. Este trabajo considera principalmente el estándar brasileño de metadatos OBAA (OBjetos de Aprendizagem baseados em Agentes) (Primo et al., 2012) para OAs el cual es una extensión del estándar IEEE-LOM enfocado a la interoperabilidad entre plataformas digital estales como TV digital, plataformas Web y dispositivos móviles. (Tramullas et al., 2013) presenta un modelo ontológico para la descripción de OAs bajo el estándar IEEE-LOM. La ontología fue denominada LOM2OWL y es almacenada en un archivo OWL. El objetivo de este trabajo es mejorar la búsqueda, reutilización y uso de OAs, tanto si éstos están organizados dentro de un repositorio específico, como si se trata de recursos dispersos en la Web.

(Vidal et al., 2010) proponen LOSO, una ontología para la búsqueda y selección de contenidos a partir del uso de reglas SWRL. La ontología fue desarrollada a partir de conocimiento descubierto aplicando técnicas en minería de datos en diferentes repositorios de OAs. La funcionalidad principal que exhibe LOSO es la clasificación de OAs de acuerdo con necesidades instruccionales. 
Todos los trabajos revisados anteriormente coinciden en la necesidad de representar los estándares de metadatos a través de un lenguaje formal para la búsqueda y recuperación de OAs. El trabajo de investigación presentado en este artículo se alinea con este enfoque, sin embargo, en el modelo propuesto se incorporan funcionalidades de planificación y evaluación personalizada que los demás modelos no consideran. Otra de las mejoras que se proponen en este artículo a los modelos actuales de computación ubicua para enseñanza-aprendizaje, es la integración de servicios de awareness así como la recomendación de recursos personalizados. Estas características de sensibilidad al contexto y alertas en los ambientes de aprendizaje disminuyen los porcentajes de deserción en cursos virtuales, debido a que mantienen la actividad cognitiva y el interés de los estudiantes.

Adicionalmente, el modelo propuesto busca extender el modelo presentado por (Duque y Ovalle, 2011) para la gestión de CVAs a través de la representación de contenidos educacionales mediante ontologías, las cuales incluyen tanto la representación de OAs como también de los perfiles de los estudiantes. Finalmente, otro objetivo de esta investigación es permitir la adaptación de los OAs no solo a las necesidades de los CVAs sino también a las preferencias de los aprendices.

\section{DESARROLLO DEL MODELO}

Esta sección presenta el proceso de concepción del SMA a partir de la metodología MAS-CommonKADS (Hasan y Isaac, 2011), el objetivo es presentar algunos modelos que ayudaron a vislumbrar los requisitos funcionales y no funcionales del sistema a partir de las fases de análisis y diseño de dicha metodología. Se decidió utilizar MAS-CommonKADS como metodología de desarrollo debido a que ofrece una gama de modelos y herramientas especializadas en la construcción de agentes de software, y en el diseño de las interacciones necesarias para la obtención de los objetivos individuales y comunes. A continuación se presentan algunos apartes de las fases de análisis y diseño del SMA, que incluyen los modelos realizados a partir de los artefactos proporcionados por AUML (Agent Unified Modeling Language).

\section{Fases de Conceptualización y Análisis del SMA}

Inicialmente se identificaron los requisitos funcionales del sistema, las necesidades y los objetivos ligados a las problemáticas actuales encontradas en la literatura. A partir de esto se construyeron los diagramas de casos de uso, que ayudaron a comprender las necesidades y a establecer las directrices para la posterior fase de análisis.

Las funcionalidades encontradas durante esta fase, contemplaban los siguientes frentes:

i) La planificación de CVA: el principal objetivo es la organización de contenidos a partir de la estructura propuesta para la construcción de los CVA. Esto permitiría guiar a los estudiantes a lo largo de los cursos, habilitando nuevos temas a medida que se avanza en los contenidos y proponiendo nuevos contenidos que les ayudarán a fortalecer el proceso de aprendizaje.

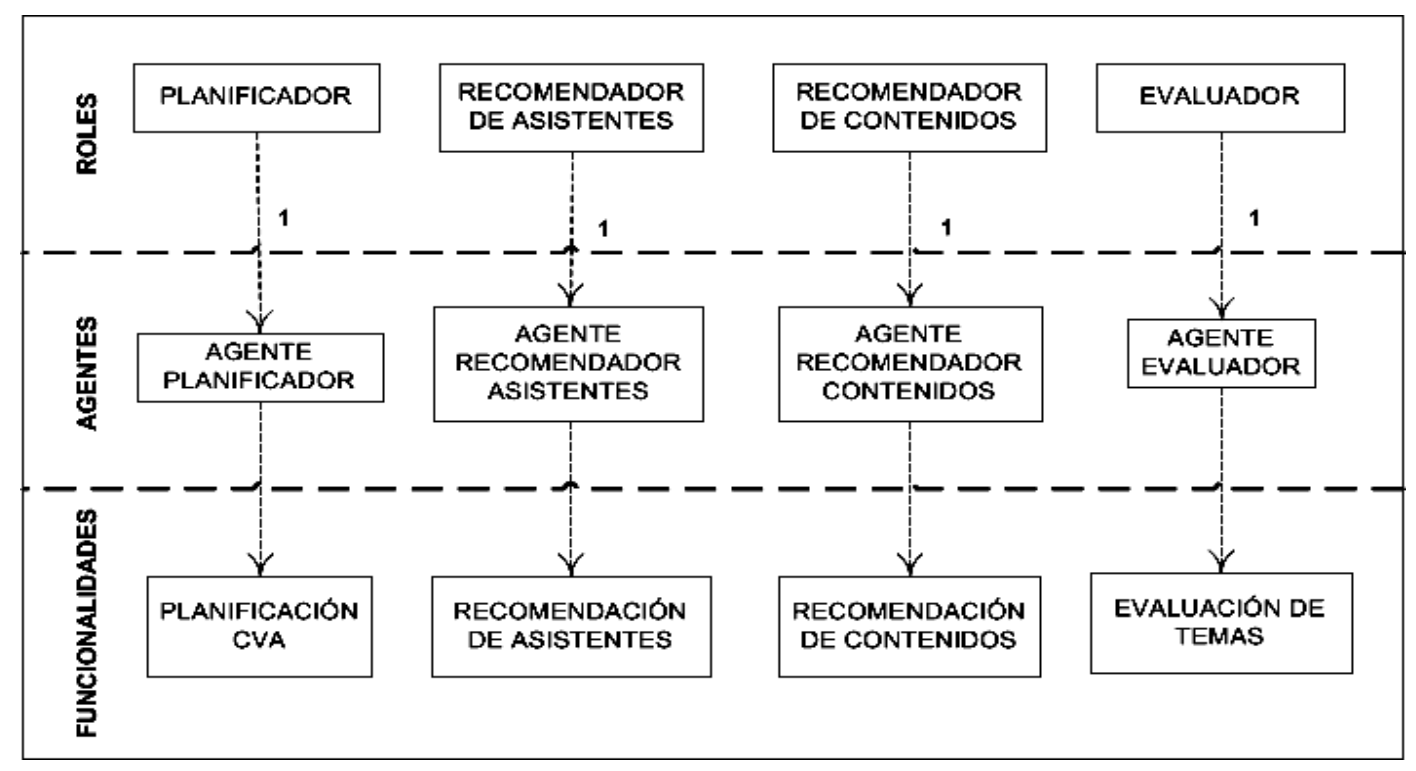

Fig. 1: Modelo de Agentes del SMA 
ii) Evaluación de contenidos: esta funcionalidad pretende evaluar el conocimiento adquirido por el estudiante con el objetivo de habilitar nuevos contenidos, de esta manera esta funcionalidad es complementaria con la planificación ya que brinda información vital relativa al avance del estudiante y permite evidenciar las falencias o fortalezas de los estudiantes por separado.

iii) Búsqueda y selección personalizada de OAs: es un componente sumamente importante dentro del sistema, puesto que ofrece contenidos personalizados que buscan despertar la concentración y el interés de los estudiantes a lo largo de los CVA, además de brindar los contenidos necesarios para el proceso de planificación. Otra característica importante que abarca esta funcionalidad es el fortalecimiento del conocimiento a lo largo de los CVA, porque en el momento en que se presenten falencias en el aprendizaje el sistema puede recomendar contenidos ajenos a la estructura de los cursos con el fin de complementar el aprendizaje y a atender dichas falencias. Estos contenidos son personalizados y adaptados a las características, gustos y limitaciones de los estudiantes, lo cual potencia el interés del estudiante y acelera el proceso de aprendizaje.

iv) Búsqueda y asignación de asistentes especializados en temáticas: permite la búsqueda y asignación de asistentes (estudiantes avanzados) de acuerdo al contexto temporal y espacial del estudiante; es decir, los asistentes serán asignados de acuerdo a la cercanía espacial a la que se encuentre con el estudiante, a los conocimientos en las áreas de interés del estudiante y a la disponibilidad de tiempo que tengan ambos.

Estas funcionalidades fueron mapeadas o asignadas a roles, lo que permitió una división inicial de tareas y objetivos. Los roles concebidos durante esta fase se asociaron a cada una de las funcionalidades, a partir de esto se consideraron los siguientes roles: planificador, recomendador de contenidos, evaluador y recomendador de asistentes.

Posteriormente y como primera actividad en la fase de análisis se mapearon los roles identificados inicialmente en agentes de software y se decidió extender un poco el modelo de agentes, añadiendo las funcionalidades del sistema con la intención de vislumbrar de mejor manera las tareas y los objetivos que tendrían asociados cada uno de estos agentes. Dicho modelo de agentes es presentado en la figura 1. Se evidencia que la relación de roles y agentes en el modelo es uno a uno, es decir, cada funcionalidad del sistema estará asociada a un único agente. De esta manera se abarcaron todos los requisitos funcionales del sistema, sin embargo, a lo largo de esta fase surgieron nuevas necesidades y requisitos no funcionales que debieron ser contemplados. Inicialmente surgió la necesidad de tener un agente que representara al estudiante dentro de la plataforma y que sirviera como intermediario entre los demás agentes y el estudiante. A partir de esta necesidad se incorporó el Agente Estudiante al sistema, con el objetivo de coordinar las actividades de los demás agentes, asociadas a un único estudiante. Esto implica que la cardinalidad de este agente es de cero a muchos $\left(0 .{ }^{*}\right)$, dicho de otra manera, existirá un Agente Estudiante por cada estudiante activo en la plataforma. Como requisito no funcional del sistema se decidió incorporar otro agente encargado de llevar a cabo servicios de awareness o de alarma, los cuales permiten generar alertas en tiempo real cuando una actividad está a punto de expirar, cuando nuevos contenidos fueron incorporados o cuando el estudiante lleva varios días sin acceder al sistema o sin realizar actividades ligadas al aprendizaje.

\section{Fase de Diseño del SMA}

Durante la fase de diseño se materializan los requisitos funcionales del sistema, se introducen nuevos requisitos no funcionales, además de aspectos técnicos que pudieron no ser considerados durante las fases previas. La figura 2 presenta el modelo propuesto para el SMA de recomendación, su distribución geográfica, los canales de comunicación entre componentes, así como las necesidades telemáticas propias de la arquitectura. Como se puede observar, la arquitectura contempla todos los agentes concebidos en las fases previas, además agrega un nuevo agente móvil. Este agente móvil aparece ante la necesidad principalmente telemática de administrar las conexiones de los dispositivos móviles de los estudiantes, en otros términos, de iniciar y/o finalizar las sesiones de los estudiantes. Otras labores de este agente son el recopilar el perfil del estudiante y almacenarlo en la base de datos, lo que obliga a utilizar la tipología de agente móvil; y obtener la información de las características del dispositivo móvil con el fin de adaptar de mejor manera la información a las especificaciones del dispositivo del estudiante.

La topología de la arquitectura muestra que el sistema es altamente distribuido a través de nodos interconectados. En el nodo principal se encuentra la plataforma principal del sistema, en la cual residen los agentes asociados a las funcionalidades principales, al igual que la base de datos del sistema. Existe un nodo plataforma móvil del estudiante por cada uno de los estudiantes activos en el sistema, allí reside el agente móvil encargado de administrar los perfiles de los estudiantes y las características de los dispositivos. 


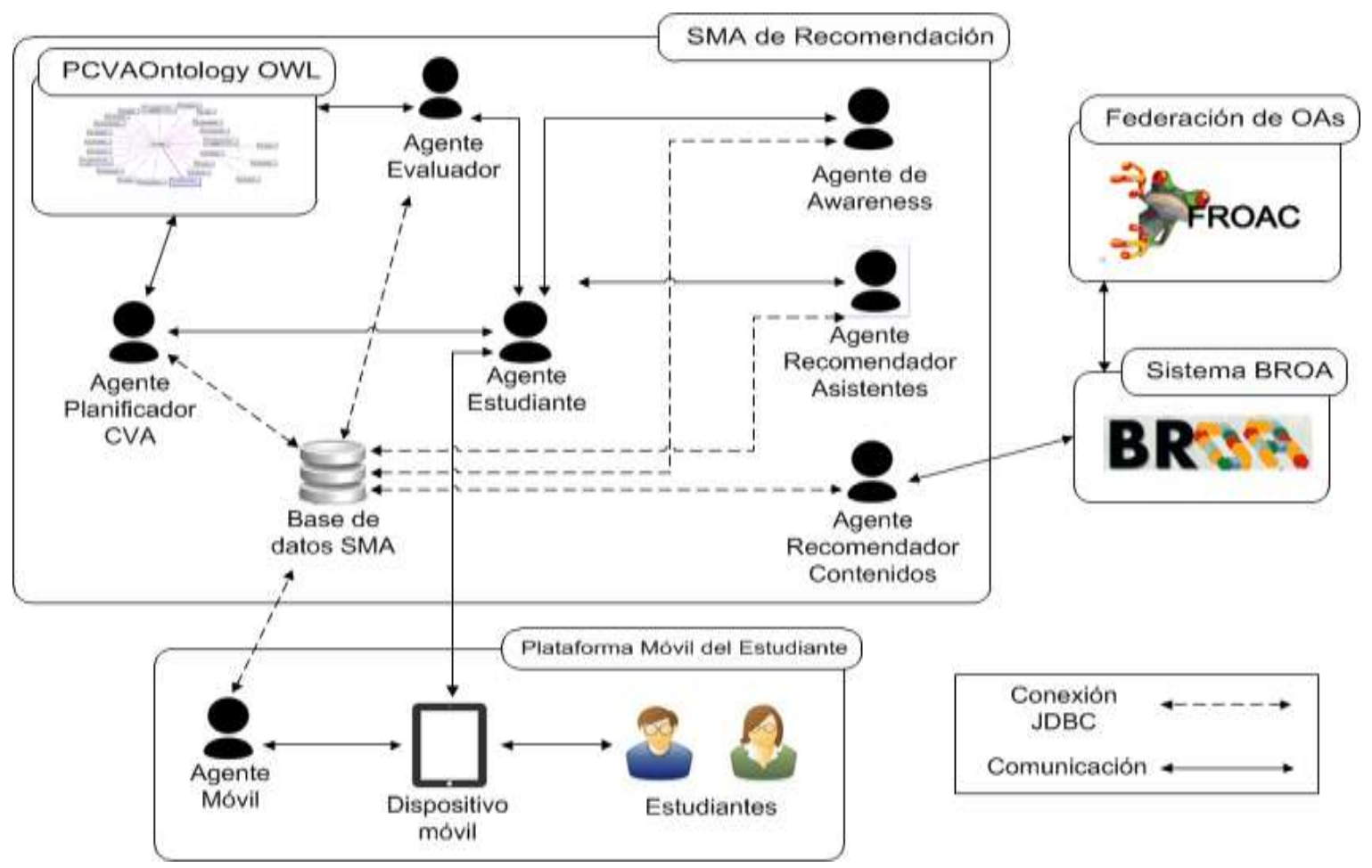

Fig. 2: Arquitectura propuesta para el SMA de recomendación personalizada

Ligado al escenario de planificación se encuentra una ontología de dominio específico, a través de la cual se infiere la estructura personalizada de los cursos virtuales a los cuales acceden los estudiantes, esta ontología denominada PCVAOntology fue concebida a partir de las fases de desarrollo propuestas por la metodología Methontology (Holanda et al., 2013), el desarrollo de dicha ontología es detallado a continuación. Finalmente el sistema cuenta con un servicio web que permite la conexión con BROA (Rodríguez et al., 2012), un SMA de búsqueda, recuperación y recomendación de OAs. Este sistema recupera OAs de repositorios y federaciones adaptados a los perfiles de los estudiantes, de igual manera detallaremos esta funcionalidad más adelante.

El proceso de diseño y desarrollo ontológico se asemeja al realizado con aplicaciones de software tradicionales, lo que implica que "no exista una manera única y correcta de modelar un dominio. El desarrollo ontológico es un proceso iterativo y los conceptos de la ontología deberán reflejar lo más fielmente posible a los objetos y relaciones del dominio" (Holanda et al., 2013).

En la actualidad, existen una gran gama de metodologías que permiten el desarrollo de ontologías a través de un conjunto de herramientas iterativas para la representación del conocimiento. Estas herramientas otorgan una documentación consistente que permite migrar, reutilizar o extender la ontología. Una de las metodologías de desarrollo más utilizadas es Methontology, la cual fue seleccionada para el desarrollo de la ontología PCVAOntology (Planificación de Cursos Virtuales Adaptativos Ontology), dado que comprende un proceso de desarrollo iterativo como se observa en la figura 3 y permite el desarrollo de ontologías a nivel de conocimiento.
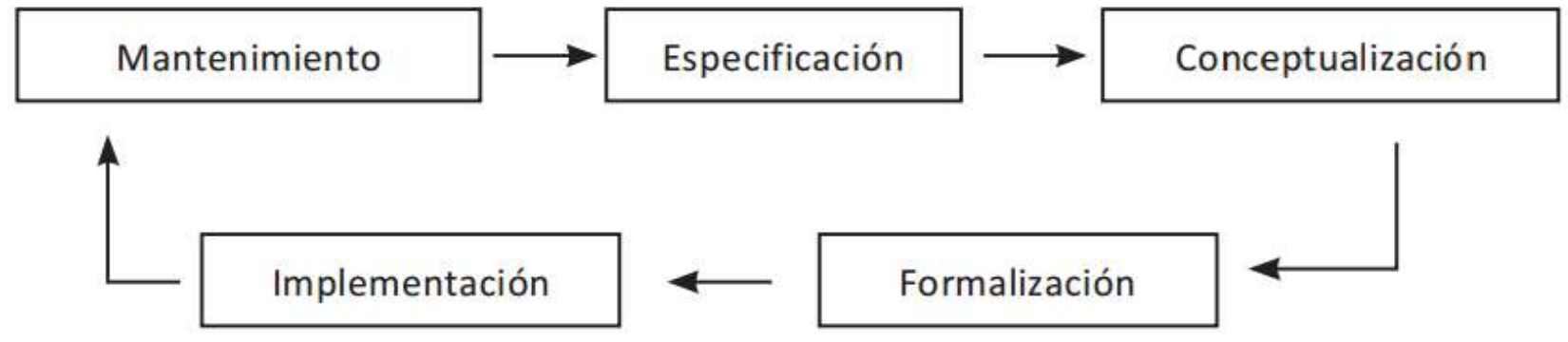

Fig. 3: Proceso de desarrollo Methontology, adaptado de (Holanda et al., 2013) 
A continuación se detallan las fases de especificación, conceptualización e implementación ontológicas.

i) Especificación ontológica: esta fase solicita al desarrollador la definición del alcance, los objetivos, el propósito, el nivel de formalidad y los usuarios finales de la ontología (Holanda et al., 2013).Para PCVAOntology se definió como alcance la generación de una descripción semántica completa que permita representar la estructura de los CVAs incluyendo el estándar de metadatos IEEE-LOM, además de considerar aspectos del perfil del usuario en cuanto a estilos de aprendizaje y características del contexto se refiere.

Los objetivos planteados comprenden el diseño de una estructura semántica que permita la construcción de una ontología para la selección de contenidos educativos virtuales, la descripción de los conceptos necesarios para definir la estructura del perfil del usuario, la definición de las reglas a través de las cuales se puedan seleccionar de forma adaptativa los contenidos para planificar el curso virtual y la adaptación de los OAs a las características del usuario y del contexto en el cual se desenvuelve.

Finalmente, se identifican como usuarios finales de la ontología a los estudiantes que podrán acceder a la información proporcionada a partir de las inferencias de la ontología, a nivel de OAs seleccionados y de los OEs alcanzados durante el curso virtual. Es importante aclarar que la ontología parte de una base de conocimientos previa, en la cual los OAs relativos a las temáticas ya han sido predefinidos, lo que se busca inferir a partir de la ontología son los recursos educativos que serán adaptados para cada tema del curso según el perfil del estudiante.

ii) Conceptualización ontológica: "la fase de conceptualización propuesta por la metodología, comprende el organizar y convertir una percepción informal de un dominio en una especificación semi-formal usando un conjunto de representaciones intermedias (tablas, diagramas) que puedan ser entendidas por los expertos del dominio y los desarrolladores de ontologías" (Holanda et al., 2013). Para esto se definen once tareas a partir de las cuales se estructura de mejor manera el conocimiento abstraído durante esta fase.

Durante esta fase se identificaron inicialmente los conceptos ligados al dominio, los cuales comprendían el conocimiento que se deseaba representar. Posteriormente se estructuró un glosario de términos que permitiera definir claramente dichos conceptos, para finalmente construir la taxonomía, donde se logró establecer las jerarquías y las relaciones existentes entre dichos conceptos. La figura 4 presenta una parte del diagrama de relaciones binarias referente a la estructura del CVA, en donde se pueden abstraer las relaciones entre las entidades del dominio consideradas.

iii) Implementación ontológica: la fase de implementación de la ontología consiste en plasmar el conocimiento identificado a partir de las fases previas a través de un lenguaje formal, en este caso se utilizó OWL mediante la herramienta Protégé (Tudorache et al., 2013). En este sentido, se crearon 23 clases, 124 instancias, 27 propiedades de tipo Objectproperties y 43 de tipo Datatypeproperties. Es importante aclarar que lo referente al estándar IEEE LOM fue mapeado en inglés para conservar la consistencia con la especificación oficial. La figura 5 presenta las jerarquías entre las clases definidas.

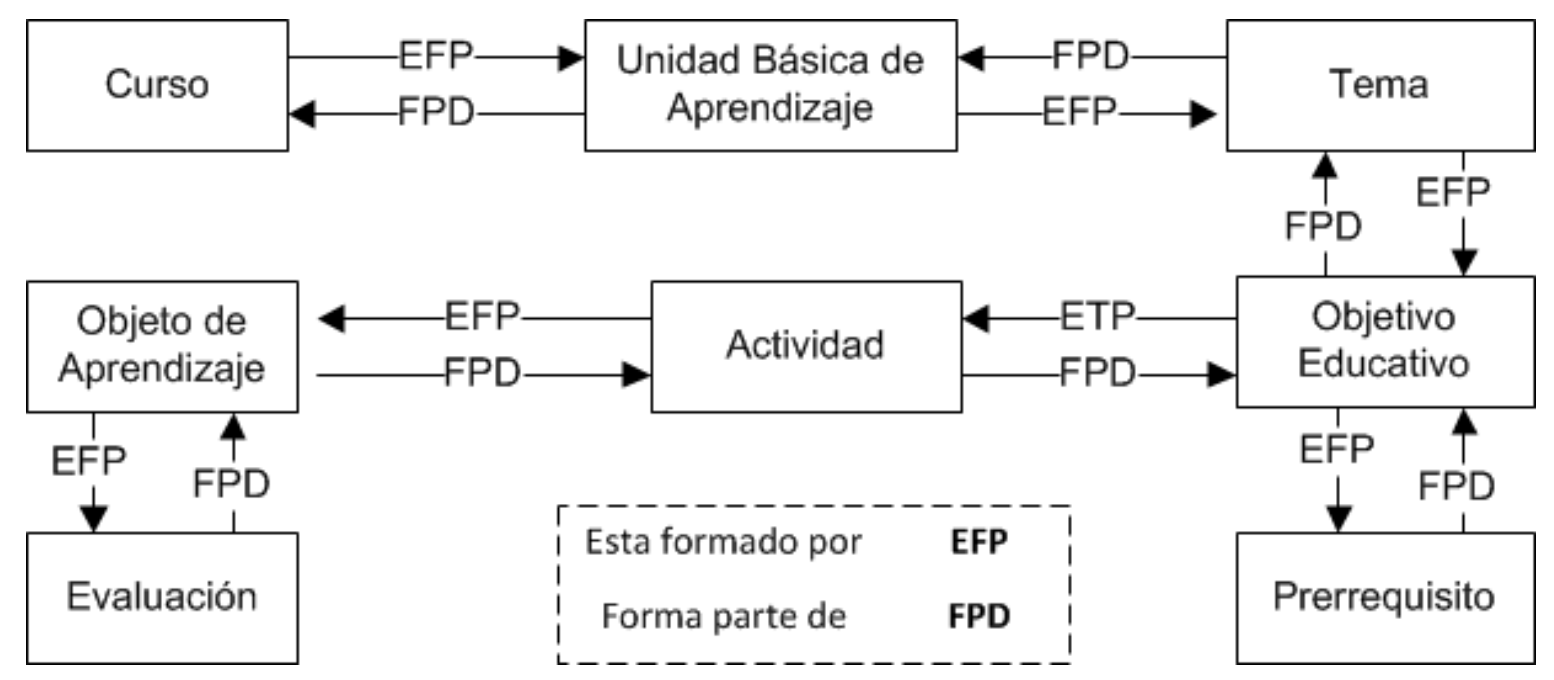

Fig. 4: Porción del diagrama de relaciones binarias referente a la estructura del CVA 
Tabla 1: Reglas SWRL para la selección de contenidos

\begin{tabular}{|c|c|}
\hline Nombre & Expresión \\
\hline ReglaDerechos & LOM(?x) $\wedge$ hasRights(?x, ?y) $\wedge$ costo(PerfilEstudiante1, ?z) $\rightarrow$ \\
ContenidoSeleccionado(?x)
\end{tabular}

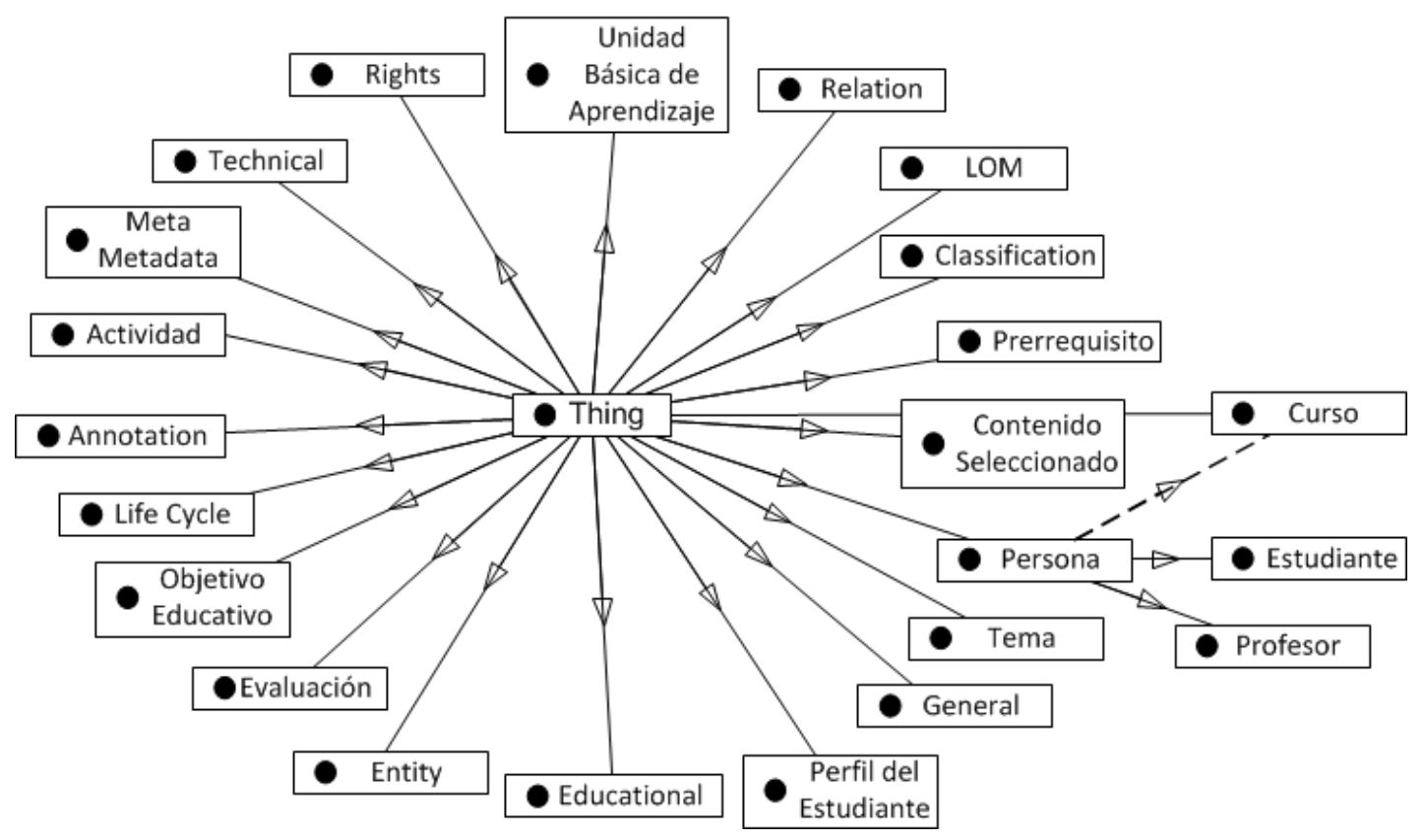

Fig. 5: Grafo de jerarquías entre clases de la ontología PCVAOntology

Para la selección de contenidos dentro de los CVA, fueron creadas cuatro reglas las cuales se presentan en la tabla 1. Para el proceso de adaptación de OAs se consideraron cuatro de las nueve categorías que propone el estándar IEEE LOM: Rights, Educational, Technical y General. Los atributos de dichas entidades fueron comparados con las características descritas en los perfiles de los estudiantes lo que permitió seleccionar exitosamente los recursos adaptados a los perfiles de usuario considerados.

\section{RESULTADOS Y DISCUSIÓN}

El sistema Multi-Agente ubicuo se implementó utilizando el Framework JADE (Bordini et al., 2013), el cual está orientado al desarrollo de SMA siguiendo los estándares FIPA (Foundation for intelligent Physical Agents) de la IEEE (IEEE, 2010). Esta característica otorga interoperabilidad a la plataforma, permitiendo así el intercambio de mensajes con sistemas que se rijan por los mismos protocolos de comunicación. Para la conexión del SMA con los dispositivos móviles fue necesario utilizar JADE-LEAP, el cual proporciona un puente de comunicación entre la plataforma de JADE a través de contenedores compatibles con ambientes móviles de JAVA bajo la tecnología J2ME-CLDC MIDP 1.0. Las configuraciones mínimas para implementar el modelo propuesto son: un servidor con sistema operativo Windows XP, procesador Intel Pentium 40 equivalente, 2 GB de RAM y una LAN. A continuación, con el fin de validar el modelo propuesto, se exhibe cada una de las funcionalidades del prototipo de SMA ubicuo que se implementó aplicado a un caso de estudio. 
Funcionalidad 1: Planificación de cursos. Como habíamos mencionado anteriormente este escenario es administrado por el Agente Planificador, quien interactúa con el Agente Recomendador de Contenidos para recuperar OAs referentes a las temáticas de los cursos. Posteriormente se recupera el perfil de estudiante almacenado en la base de datos del sistema, y luego se mapea toda esta información en la ontología de descripción semántica del conocimiento asociado a los CVAs. Esta ontología, denominada PCVAOntology y especificada en lenguaje OWL, fue desarrollada utilizando Protégé y permite la selección de recursos educativos asociados a los CVAs a partir de reglas SWRL. Para la representación de la estructura de los OAs dentro de la ontología, se utilizó el estándar IEEE-LOM considerando las relaciones jerárquicas que este plantea y sus cardinalidades. Pensando en facilitar el proceso de selección de OAs, fue necesario también extender el modelo propuesto por (Duque y Ovalle, 2011) para la representación de la estructura de los CVAs. Este modelo propone una estructura jerárquica en donde los cursos virtuales se descomponen en Unidades Básicas de Aprendizaje, las que a su vez se desglosan en temas. Los temas tienen objetivos educativos, los cuales necesitan cumplir ciertos prerrequisitos para poder ser accedidos. Finalmente, cada tema tiene esquematizadas unas actividades con sus respectivos OAs para el aprendizaje.

En la figura 6 se presenta la interfaz de navegación a través de los CVAs asociados al estudiante, allí se pueden seleccionar los cursos que se desean acceder, el estado del estudiante dentro del curso, los recursos seleccionados a partir de proceso de planificación para cada temática y los asistentes especializados en las temáticas de los cursos. Estos asistentes corresponden a los seleccionados previamente por el Agente Recomendador de Asistentes, quien ofrece también un canal de comunicación estudiante-asistente, que permite la interacción entre ambos.

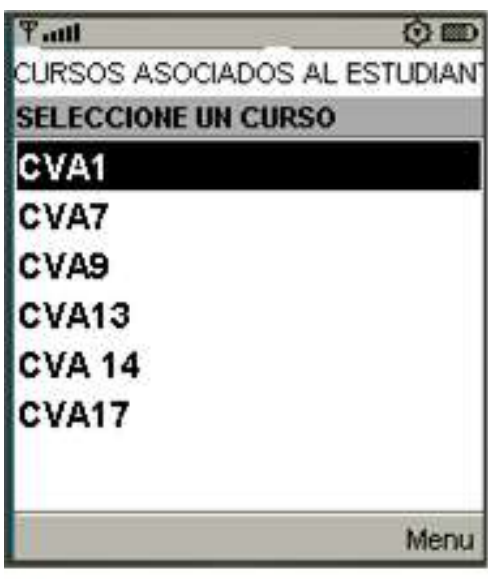

(a) Selección de cursos

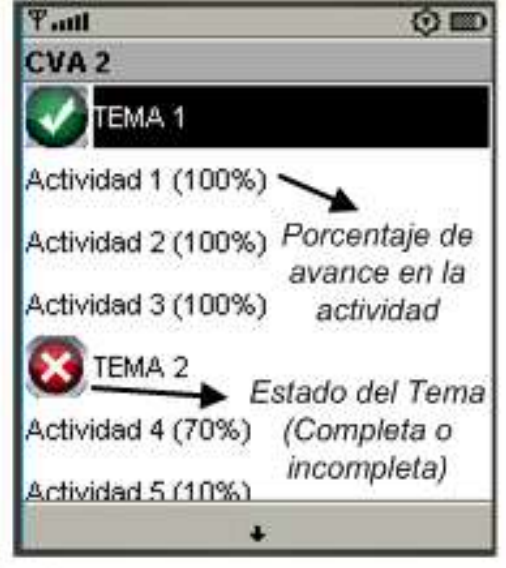

(b) Estado del estudiante en Ios CVA

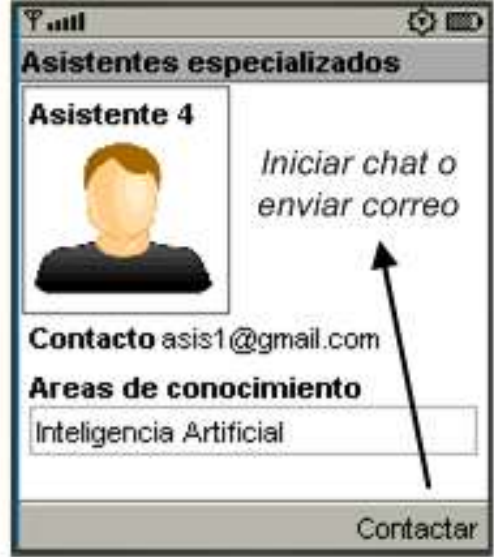

(c) Recomendación de Asistentes especializados

Fig. 6: Escenario de planificación

Funcionalidad 2: Evaluación de temas. Este escenario es puesto en marcha en el momento en que el usuario concluye un tema, o desea exonerarse del mismo. Las preguntas son seleccionadas de un banco de preguntas previamente constituido y asociado a cada uno de los temas de los CVA. De acuerdo a la estructura de evaluación propuesto por (Duque y Ovalle, 2011), por cada pregunta hay una o varias respuestas y son validadas a través de un campo bivaluado (1 si la respuesta es correcta y 0 si es incorrecta). Otro de los parámetros más importantes es el tiempo medio de respuesta necesario para que el estudiante pueda responder a la pregunta y el tipo de pregunta que describe si la pregunta es de respuesta múltiple, única elección, verdadero-falso, etc.

Funcionalidad 3. Servicios de Awareness. Esta funcionalidad permite generar alarmas en cualquier momento de ejecución del sistema. El Agente de Awareness está encargado de llevar a cabo comportamientos cíclicos que permitan estar monitoreando las actividades del estudiante en la base de datos, con el fin de mantenerlo informado de las actividades en tiempo real. Estas alarmas pueden estar relacionadas con actividades cuyo tiempo de caducidad está por cumplirse o simplemente con invitaciones que ayuden a disminuir los porcentajes de deserción de los cursos (ver figura 7 (c)).

Funcionalidad 4. Servicios de Recomendación de Recursos Educacionales. Este escenario contempla características proactivas por parte del Agente Recomendador de Contenidos, ya que ofrece nuevos recursos virtuales educativos (OAs) en caso de que el estudiante presente falencias en la evaluación de los temas o si este decide solicitarlos. 


\begin{tabular}{|l|}
\hline P...tII \\
\hline Pregunta 1 \\
\hline $\begin{array}{l}\text { Selección múltiple Cúal de las } \\
\text { siguientes opciones no es una } \\
\text { campo de aplicación de la IA }\end{array}$ \\
\hline Respuestas \\
Sistemas Multi-Agente \\
Lógica Difusa \\
Investigación de Operaciones \\
Visión Artificial \\
\hline \\
\hline
\end{tabular}

(a) Evaluación de temas

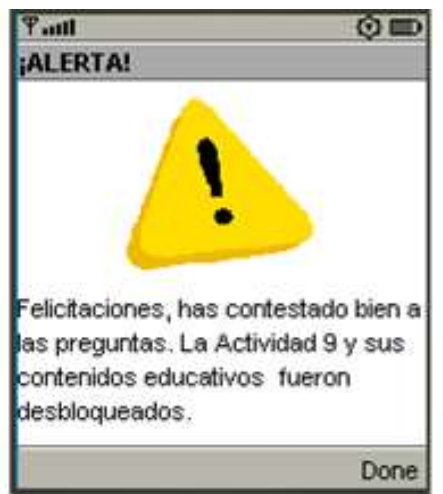

(b) Habilitación de contenidos

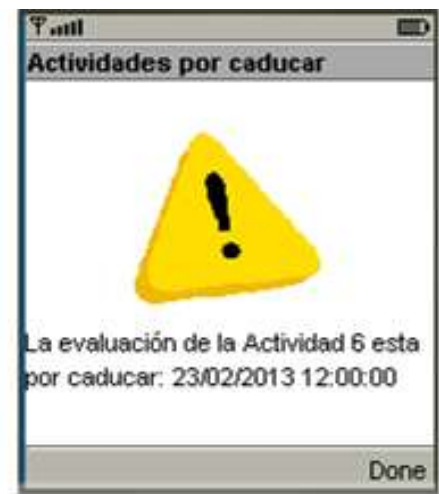

(c) Servicios de Awareness

Fig. 7: Escenario de evaluación

La validación realizada a partir del caso de estudio planteado fue desarrollada a partir de la versión Split Stand-alone de JADE-LEAP, la cual consiste en dividir la plataforma de agentes en dos componentes; un Front-End, el cual se despliega en el dispositivo mediante un MIDlet, y otro Back-End desplegado en el servidor, en el cual son llevadas a cabo las inferencias y los cálculos robustos. La base de datos del sistema fue implementada con el sistema de gestión de base de datos MySQL y las inferencias ontológicas fueron abstraídas mediante el Framework JENA desde el archivo OWL generado por Protégé.

Se realizaron pruebas de estrés al servidor conectando alrededor de 20 dispositivos móviles caracterizados por la configuración J2ME - CLDC MIDP 1.0; los cuales fueron desplegados y simulados mediante el Toolbox Sun Java Wireless Toolkit. La plataforma fue desplegada en un servidor con sistema operativo Windows 7, cuatro GB de memoria RAM y procesador Intel core i3. A partir de estas características, se logró corroborar las grandes ventajas que trae el utilizar el SMA, ya que al dividir las tareas en diferentes nodos los tiempos de respuesta se reducen y la información es entregada de manera más ágil.

La validación realizada a partir de un escenario planteado muestra que los resultados son bastante satisfactorios, presenta un sistema en donde los cálculos robustos y las inferencias son llevados a cabo en el servidor y los dispositivos móviles únicamente se encargan de generar las interfaces. Se realizaron pruebas de estrés al servidor conectando una gran cantidad de dispositivos móviles para ver como se comportaba el sistema, a partir de esto se logró corroborar las grandes ventajas que trae el utilizar SMA, ya que al dividir las tareas en diferentes nodos los tiempos de respuesta se reducen y la información es entregada de manera más ágil.

En cuanto a la validación de las recomendaciones otorgadas por el sistema a partir de las inferencias obtenidas con las reglas SWRL planteadas, fueron seleccionados veinte OAs con sus respectivos metadatos. Los OAs fueron seleccionados con base en las categorías consideradas para la aplicación de las reglas SWRL, esto con el fin de que la información consignada en los metadatos fuera diversa y poder observar de mejor manera que tan bien se acoplaban a los perfiles de estudiantes. Fueron considerados 3 perfiles de estudiantes con diferentes características tales como dificultad de los contenidos, niveles de interactividad, tipos de recurso, estilos de aprendizaje, duración y costos de los recursos. A partir de estas características y las descripciones de los OAs expuestas en los metadatos, fueron realizadas las inferencias y las recomendaciones arrojadas por el sistema fueron muy satisfactorias. Para evaluar la efectividad del modelo propuesto y del SMA como tal, se consideraron tres estudiantes quienes de una lista predefinida, seleccionaron los OAs que más se acoplaban a sus características, gustos y limitaciones, obteniendo de esta manera los resultados que se presentan en la tabla 2.

Esta investigación ha permitido la materialización de un modelo de SMA Ubicuo de recomendación personalizada para dispositivos móviles, brindando una posible solución a las necesidades actuales referentes a los métodos de enseñanza-aprendizaje virtual. Es importante aclarar que la representación ontológica que se plantea en la investigación, no abarca todas las categorías que considera el estándar IEEE-LOM, de igual manera los perfiles de los estudiantes no contemplan información referente al contexto temporal y/o espacial del estudiante; estos elementos quedan planteados como trabajo futuro y extienden el marco de la investigación. Otra mejora importante que podría considerar el SMA, es la migración a una plataforma móvil mucho mas estandarizada, la cual soporte dispositivos con mejores herramientas y características. Esta plataforma móvil podría ser Android, la cual permite el aprovechamiento de características propias de los Smartphone que pueden ser de utilidad al momento de recuperar información referente al contexto del usuario, como por ejemplo el GPS y las redes sociales. 
Tabla 2: Resultados obtenidos a partir de la ontología

\begin{tabular}{|c|c|c|c|c|}
\hline & OAs Considerados & $\begin{array}{c}\text { OAs seleccionados por el } \\
\text { estudiante }\end{array}$ & $\begin{array}{c}\text { OAs recomendados por el } \\
\text { sistema para el CVA }\end{array}$ & $\begin{array}{c}\text { Porcentaje de } \\
\text { acierto }\end{array}$ \\
\hline Perfil 1 & 20 & 5 & 4 & $80 \%$ \\
\hline Perfil 2 & 20 & 7 & 5 & $71,4 \%$ \\
\hline Perfil 3 & 20 & 8 & 5 & $62,5 \%$ \\
\hline
\end{tabular}

\section{CONCLUSIONES}

A partir del modelo propuesto, del prototipo de SMA construido y de los resultados obtenidos del proceso de validación, se pueden obtener las siguientes conclusiones:

i) Se ha presentado un modelo de recomendación de contenidos educativos que comprende diversas funcionalidades, las cuales están orientadas al mejoramiento del proceso de aprendizaje personalizado de los estudiantes registrados en CVAs.

ii) El modelo ha permitido integrar diferentes herramientas para la selección de contenidos, la planificación de cursos, la evaluación temática y la generación de servicios de awareness a partir de plataformas móviles que brindan al SMA características de ubicuidad.

iii) El modelo facilita la representación de las estructuras de conocimiento asociadas a los CVAs, así como el estándar de etiquetado de metadatos IEEE-LOM y los perfiles de estudiante a través de un lenguaje formal como OWL, lo que garantiza y permite la incorporación de SMA adaptativos a la creciente Web Semántica.

iv) La investigación realizada propone una arquitectura para la planificación automática de CVAs basada en agentes inteligentes la cual pretende el aprovechamiento de las características de interoperabilidad de ontologías descritas en OWL para la abstracción de conocimiento. Todo lo anterior a partir de la integración con el lenguaje de programación JAVA.

v) Las reglas SWRL propuestas para la selección de OAs dentro de las actividades del CVA, seleccionaron exitosamente recursos adaptados a los perfiles de usuario considerados.

vi) Los resultados obtenidos muestran que el uso de este tipo de tecnologías favorece la adquisición del conocimiento por parte de los estudiantes y brinda mecanismos que despiertan el interés de éstos, proporcionando un ambiente de aprendizaje continuo y ubicuo, es decir, accesible en cualquier momento y lugar. De esta forma la evolución de este modelo de aprendizaje podría ser utilizado por las empresas en sus procesos de capacitación, al mismo tiempo que permite realizar un seguimiento personalizado en cuanto al aprendizaje que cada empleado recibe durante la formación empresarial específica.

\section{AGRADECIMIENTOS}

Esta investigación fue financiada parcialmente por el proyecto de investigación: "Mejoramiento de la capacidad académica, visibilidad, contacto e interacción con la comunidad nacional e internacional del Grupo de Investigación en Inteligencia Artificial de la Universidad Nacional de Colombia" - Convocatoria Nacional, Modalidad 2, 2012-2013, con código QUIPU 202010010368.

\section{REFERENCIAS}

Acevedo, C., Arciniegas, J.L., García, X. y Perrinet, J., Proceso de Adaptación de una Aplicación de eaprendizaje a t-aprendizaje. Información Tecnológica, 21(6), 27-36 (2010).

Berners-Lee, T., Hendler, J. y Lassila, O., The Semantic Web. Scientific American, 284(5), 28-37 (2001).

Bordini, R., Braubach, L., Dastani, M., El Fallah Seghrouchni, A., Gomez-Sanz, J., Leite J., O'Hare, G., Pokahr, A. y Ricci, A., A Survey of Programming Languages and Platforms for Multi-Agent Systems Informatica, 30(33), 33-44 (2006).

Charitonos, K., Blake. C., Scanlon, E., y Jones, A., Museum learning via social and mobile technologies: (How) can online interactions enhance the visitor experience? British Journal of Educational Technology, 43(5), 802-819 (2012).

Chen, Ch. y Huang, T., Learning in a u-Museum: Developing a context-aware ubiquitous learning environment. Computers \& Education Journal, 59, 873-883 (2012). 
Duque, N. y Ovalle, D., Artificial intelligence planning techniques for adaptive virtual course construction, Dyna, 78(170), 70-78 (2011).

Gómez, S., Zervas, P., Sampson, D. G. y Fabregat, R., Context-aware adaptive and personalized mobile learning delivery supported by UoLmP. Journal of King Saud University - Computer and Information Sciences, 26(1), 47-61 (2014).

Hwang, G., Yang, T., Tsai, C. y Yang, S., A context-aware ubiquitous learning environment for conducting complex science experiments. Computers \& Education Journal, 53, $402-413$ (2009).

Holanda, O., Isotani, S., Bittencourt, I. I., Elias, E. y Tenório, T., JOINT: Java ontology integrated toolkit. Expert Systems with Applications, 40(16), 6469-6477 (2013).

Hasan, S. y Isaac, R., An integrated approach of MAS-CommonKADS, Model-View-Controller and web application optimization strategies for web-based expert system development. Expert Systems with Applications, 38(1), 417-428 (2011).

Kinshuk, D. P. y Graf, D. S., Ubiquitous Learning, en Encyclopedia of the Sciences of Learning, P. D. N. M. Seel, Ed. pp. 3361-3363, Springer US (2012).

Liu, T. y Jiang, Z., Distributed nonlinear control of mobile autonomous multi-agents. Automatica, 50(4), 10751086 (2014).

Meditskos, G. y Bassiliades, N., A Rule-Based Object-Oriented OWL Reasoner. IEEE Transactions on Knowledge and Data Engineering, 20(3) 397-410 (2008).

Primo, T., Silva, J.L.T., Ribeiro, A.M., Vicari, R.M. y Boff, E., Towards Ontological Profiles in Communities of Practice. IEEE Multidisciplinary Engineering Education Magazine, 7, 13-22 (2012).

Primo, T., Behr, A. y Vicari, R.M., A Semantic Web Approach to Recommend Learning Objects», en Highlights on Practical Applications of Agents and Multi-Agent Systems, J. M. Corchado y otros 8 editores, pp. 340-350, Springer, Heidelberg (2013).

Raju, P. y Ahmed, P., Enabling technologies for developing next-generation learning object repository for construction, Automation in Construction, 22, 247-257 (2012).

Rodríguez, P., Tabares, V., Duque, N., Ovalle, D. y Vicari, R, Multi-agent Model for Searching, Recovering, Recommendation and Evaluation of Learning Objects from Repository Federations. Iberamia, Springer, Heidelberg, 631-640 (2012).

Shoham, Y. y Leyton-Brown K., Multiagent systems: algorithmic, game-theoretic, and logical foundations. Cambridge University Press (2008).

Tudorache, T., Nyulas, C. I., Noy, N. F., y Musen, M. A. WebProtégé: A Collaborative Ontology Editor and Knowledge Acquisition Tool for the Web. Semantic Web Journal, 4(1) 89-99 (2013).

Tramullas, J., Sánchez-Casabón, A., Garrido-Picazo, P., An Evaluation based on the Digital Library user: An Experience with Greenstone Software. Procedia - Social and Behavioral Sciences, 73,167-174 (2013).

Vidal, C., Segura, A., Menéndez, V., Zapata, A., Prieto, M., Metadata and Ontologies in Learning Resources Design, en Knowledge Management, Information Systems, E-Learning, and Sustainability Research, Communications in Computer and Information Science, 111, 105-114, Springer, Heidelberg (2010).

Vojak, C., Kline, S., Cope, B., McCarthey, S. y Kalantzis, M., New Spaces and Old Places: An Analysis of Writing Assessment Software. Computers and Composition. 28(2) 97-111 (2011).

Wang, S. y Wu, Ch., Application of context-aware and personalized recommendation to implement an adaptive ubiquitous learning system. Expert Systems with Applications, 38, 10831-10838 (2011).

Wooldridge, M., An introduction to multi-agent systems. Second edition, Wiley Publishers. United Kingdom (2009). 